\title{
Modulation of Apoptosis During HTLV-1-Mediated Immortalization Process In Vitro
}

\author{
Claudia Matteucci, ${ }^{\mathbf{1}}$ Emanuela Balestrieri, ${ }^{2}$ Beatrice Macchi, ${ }^{3}$ and Antonio Mastino ${ }^{2 *}$ \\ ${ }^{1}$ Department of Experimental Medicine and Biochemical Science, University of Rome “Tor Vergata," Rome, Italy \\ ${ }^{2}$ Department of Microbiological, Genetic and Molecular Sciences, University of Messina, Messina, Italy \\ ${ }^{3}$ Department of Neuroscience, University of Rome "Tor Vergata," Rome, Italy
}

Suppression of apoptosis has been proposed as a mechanism involved in the transforming action of human T-cell leukemia/lymphotropic virus type-1 (HTLV-1). However, there is evidence that HTLV-1 and its protein Tax also induce apoptosis. To resolve this apparent paradox, apoptosis was monitored in primary cultures of peripheral blood lymphocytes (PBLs) from healthy donors, following HTLV-1 infection in vitro. High levels of apoptosis in HTLV-1 infected cultures during the first weeks after infection were detected. Apoptosis was not related to the presence of uninfected cells, as revealed by a fluorescence in situ hybridization assay. Successively, a progressive decrease in apoptosis in infected cultures going towards immortalization, was observed. When IL-2 in the medium was replaced by IL-4, allowing the cells to be efficiently infected by HTLV-1 but not immortalized, apoptosis levels tended to increase, instead of decreasing, with the ongoing time. The caspase cascade was remarkably activated in PBLs recently infected in vitro by HTLV-1, but apoptosis was only partly reduced by caspase inhibitors. Even if spontaneous apoptosis was relatively low in long-term cultures of PBLs immortalized by HTLV-1 in vitro, Fas death-receptor expression and function were well conserved. These observations provide a new rationale for explaining the dual effect of HTLV-1 in controlling apoptosis. J. Med. Virol. 74:473-483, 2004. ๑ 2004 Wiley-Liss, Inc.

KEYWORDS: adultT-cell leukemia/lymphoma; viral oncogenesis; cell death; Fas; caspases; fluorescence in situ hybridization

\section{INTRODUCTION}

Two of the key hallmarks of transformed cells are the acquired capabilities to evade apoptotic cell death and to proliferate limitlessly [Hanahan and Weinberg, 2000]. As a consequence, it seems reasonable that oncogenesis promoted by viral agents implies the interference of viral components with host cell genes which regulate death and growth cell machineries. The human T-cell leukemia/lymphotropic virus type-1 (HTLV-1) is a retrovirus etiologically associated to adult T-cell leukemia/ lymphoma as well as to degenerative disorders known as HTLV-1 associated myelopathy/tropic spastic paraparesis [Barmak et al., 2003]. It is known that adult T-cell leukemia/lymphoma is characterized by a remarkable monoclonal expansion of malignant T-cells, preferentially showing the CD4+ phenotype, harboring integrated proviral virus. This is preceded by a premalignant phase of oligo/polyclonal expansion of non-malignant HTLV-1 infected cells [Cavrois et al., 1996]. Mechanisms involved in the shift from the premalignant to the malignant phase of HTLV-1 infection have not been elucidated. Existing data suggest that oncogenic transformation caused by HTLV-1 is a complex event arising from a multistep process in which the virus, through the action of specific viral gene products acting on regulatory cellular genes, induces T-cell proliferation. This results both in the accumulation of genetic defects and in the dysregulation of growth and death in infected cells [Mortreux et al., 2003]. HTLV-1 encodes several regulatory proteins. Among these, Tax seems the main protein responsible for in vivo transformation of HTLV1 infected cells, either by directly causing activation and proliferation of infected cells, independently of antigenic stimulation, or by increasing chromosomal abnormalities and mutation rates within the infected cells [Maruyama et al., 1990; Miyake et al., 1999]. In

Grant sponsor: Istituto Superiore di Sanità, AIDS Projecte; Grant sponsor: University of Rome "Tor Vergata" (to B.M.); Grant sponsor: Italian Ministry of Education, University and Research, Research Projects of National Interest; Grant sponsor: University of Messina (to A.M.).

*Correspondence to: Prof. Antonio Mastino, PhD, Department of Microbiological, Genetic and Molecular Sciences, University of Messina, Salita Sperone 31, 98166 Messina, Italy.

E-mail: mastino@med.uniroma2.it

Accepted 20 May 2004

DOI 10.1002/jmv.20201

Published online in Wiley InterScience

(www.interscience.wiley.com) 
addition, it was shown that cells from adult T-cell leukemia/lymphoma patients do not undergo spontaneous apoptosis in vitro after short-term culture in the presence of IL-2 [Tsuda et al., 1993]. Recent reports have shown that resistance to apoptosis in HTLV-1-infected leukemic cells may be due to autocrine antiapoptotic signaling provided by over-expression of chemokines [Ruckes et al., 2001]. Nevertheless, it has been reported that adult T-cell leukemia/lymphoma cells, which express Fas antigen, retain a certain susceptibility to Fas-mediated apoptosis [Maeda et al., 1999]. Taken together these data indicate that in adult T-cell leukemia/lymphoma patients, leukemogenesis is associated to a rescue of host cells from death. Despite the evidence for virus-induced inhibition of cell death during transformation by HTLV-1, some results suggest that the issue might not be so simple. In fact, conflicting results have shown that the viral protein Tax, in addition to exerting expected anti-apoptotic effects, also enables HTLV-1-infected cells to undergo apoptosis, under certain experimental conditions. For example, the expression of Tax in an inducible system promoted rather than inhibited cell death [Chlichlia et al., 1995]. This Tax-triggered death was critically dependent on ICEprotease function [Chlichlia et al., 1997] and associated with a marked up-regulation of the Fas ligand (FasL) gene [Chen et al., 1997]. It has been hypothesized that induction of apoptosis might depend on nuclear expression of the CREB-binding protein (CBP)/p300binding domain of the HTLV-1 transactivator Tax [Nicot and Harrod, 2000]. Conversely, it has been shown that HTLV-1-expressing human T-cell lines showed reduced susceptibility to anti-Fas [Copeland et al., 1994], and to $\mathrm{TNF} \alpha$ stimulation [Yang et al., 2002]. This resistance to apoptosis might be due to the activation of NF-kB via Tax protein in HTLV-1 infected cells [Kawakami et al., 1999], to Tax-induced expression of anti-apoptotic genes [Brauweiler et al., 1997; Mori et al., 2001] and should involve p21 [Kawata et al., 2003]. In addition, Tax might ensure protection against apoptosis in the presence of various stress-inducing agents [Torgeman et al., 2001]. Relevant to this work it should be noted that all the experimental approaches concerning the capability of HTLV-1 and, in particular, of Tax to regulate apoptotic cell death did not consider time as a variable which could influence this dual, regulatory action. Thus, the apparent paradox of the opposite effects of Tax in controlling cell death prompted us to investigate whether during the natural progression of HTLV-1 infection induction of or resistance to apoptosis could prevail depending on timing. Advantage was taken in this study of the availability of the experimental model of HTLV-1 infection in vitro which allowed a longitudinal study to detect apoptosis levels at various times following infection.

\section{MATERIALS AND METHODS}

\section{Cells and Infection}

Human peripheral blood mononuclear cells from healthy donors, serum negative for HIV and hepa- titis $\mathrm{B}$ and $\mathrm{C}$, were separated by density gradient (Lympholyte-H, Cedarlane, Hornby, Ontario, Canada) according to the standard technique. Freshly recovered suspensions of peripheral blood mononuclear cells, contained $12-20 \%$ monocytes, $9-16 \%$ NK cells, and $2-8 \% \mathrm{~B}$ lymphocytes as defined by expression of CD14, CD16, and CD19 molecules, respectively. However, non-T-cells were practically absent after 1 week in culture. In this study, these cells in culture are referred to as peripheral blood lymphocytes (PBLs). After washing in PBS, a total of $1 \times 10^{7} \mathrm{PBLs}$, at an initial concentration of $1 \times 10^{6} \mathrm{per}$ $\mathrm{ml}$, were co-cultured with lethally irradiated MT-2 cells (120 Cy, from a caesium gamma cell irradiator 1000, Canada Atomic Energy Ltd., Canada), at an acceptor:donor ratio of 5:1. MT-2 is a HTLV-1-transformed cell line derived from cord blood mononuclear cells infected in vitro with the virus from an adult T-cell leukemia/ lymphoma patient [Miyoshi et al., 1981]. In some experiments, CD4+ separated T-cells were infected with HTLV-1 by the same method used for total PBLs. CD4+ T-cells were separated through immunomagnetic beads conjugated with specific CD4 mAb (Dynabeads, Oslo, Norway) as described previously [Macchi et al., 1993]. Briefly, PBLs were mixed with CD4-conjugated immunomagnetic beads and incubated at $4{ }^{\circ} \mathrm{C}$ for $30 \mathrm{~min}$ on a rotating wheel. The cells adherent to the beads were separated from the other cells using a magnet (Dynabeads). CD4+ cells were detached from the beads through incubation with mouse IgG Detachbead (Dynabeads) for $30 \mathrm{~min}$ at room temperature, according to the manufacturer's instructions. Co-cultures of PBLs and irradiated MT-2 cells were grown in RPMI 1640 medium supplemented with $20 \%$ FCS, glutamine, penicillin-streptomycin (Gibco-Invitrogen Co., Paisley, Scotland, UK). Human recombinant IL-2 (Chiron, Emeryville, CA) or, in some experiments, human recombinant IL-4 (kindly provided by Dr. Narula, ScheringPlough, Kenilworth, NJ) were added weekly to culture medium at final concentrations of $20 \mathrm{U} / \mathrm{ml}$ or of $15 \mathrm{ng} / \mathrm{ml}$, respectively. Cultures underwent weekly passage. At each passage, cell growth was monitored by evaluating living cells, using the trypan blue dye exclusion test, and cell concentration was readjusted at $8 \times 10^{5} / \mathrm{ml}$ living cells, as previously described [Mastino et al., 1997]. Results of cell growth were expressed as total cell number, calculated theoretically weekly as the total cell number calculated at the previous week multiplied by the number of cells, expressed as millions of viable cells per milliliter, detected before weekly adjustment. For the first week, the total cell number was the actual total number of cells detected before the first adjustment. Aliquots of irradiated MT-2 cells, utilized as donor cells for infection and kept separately in culture medium for the duration of the experimental culture, showed no evidence of growth.

\section{Apoptosis, PCR, and Protein Analyses}

To assess the percentage of apoptotic cells, the HTLV1-infected cell lines were monitored every 2 weeks. 
Aliquots from cell lines were collected and centrifuged according to the standard density gradient technique to eliminate debris and dead cells. Residual apoptosis in cell cultures soon after centrifugation was in any case less than $10 \%$. After washing in PBS the cells were adjusted at a density of $1 \times 10^{6} \mathrm{cell} / \mathrm{ml}$, with the addition of IL-2 or, in some experiments, IL-4, and were cultured for an additional $24 \mathrm{hr}$ before they were washed and prepared for apoptosis, PCR, and protein analysis. Agonist, anti-human Fas mAb (clone CH11, 500 ng/ml; Upstate Biotechnology, Lake Placid, NY) or caspase inhibitors, Z-VAD-FMK and Z-DEVD-FMK (Calbiochem, San Diego, CA) diluted in DMSO (Sigma, St Louis, MO), were added to some replicate cultures during the last $24 \mathrm{hr}$ of incubation time. In all samples, apoptosis was evaluated by flow cytometry analysis of isolated nuclei following DNA staining with propidium iodide, using a method which distinguishes apoptosis from necrosis and viability [Matteucci et al., 1999]. Briefly, cells were lysed and stained with a solution containing $2 \%$ Triton $\mathrm{X}-100,25 \mu \mathrm{g} / \mathrm{ml}$ propidium iodide, and $0.05 \%$ sodium citrate (all from Sigma). Isolated nuclei were then analyzed by using a FACScan flow cytometer (BD Biosciences, Immunocytometry Systems, Mountain View, CA). In some samples, direct analysis of DNA fragmentation was carried out at single-cell level using the TUNEL technique. Labeling of strand breaks with fluorescein-dUTP by terminal deoxynucleotidyl transferase was carried out, using a commercial kit (Roche Diagnostics, Mannheim, Germany), according to the manufacturer's instructions. Analysis of the labeled cells was performed by flow cytometry following adequate gating by scatter parameters to exclude cell debris and large cell aggregates, and to include presumably apoptotic, necrotic and living cells. For each sample, 5,000 events were acquired. For DNA-PCR analysis, $5 \times 10^{6}$ cells were incubated with proteinase $\mathrm{K} 50 \mu \mathrm{g} / \mathrm{ml}$ and SDS $0.5 \%$ at $37^{\circ} \mathrm{C}$ and DNA was extracted in phenolchloroform-isoamylalcohol (50:49:1), according to standard procedures (all from Sigma). One microgram of DNA was used as a template and was amplified in a standard PCR reaction mix: $1 \times$ PCR buffer, $0.2 \mathrm{mM}$ dNTPs, $0.5 \mu \mathrm{M}$ primer pair specific for the pol region (SK54, SK56, Perkin Elmer, Norwalk, CT) of HTLV-1 and 1.25 U of Taq polymerase (Roche Diagnostics). Samples were subjected to 30 cycles of PCR amplification, each cycle consisting of $30 \mathrm{sec}$ at $94^{\circ} \mathrm{C}$ (DNA denaturation), $30 \mathrm{sec}$ at $55^{\circ} \mathrm{C}$ (primer annealing), and $45 \mathrm{sec}$ at $72^{\circ} \mathrm{C}$ (primer extension) on a Cetus DNA thermal cycler 2400 (Perkin Elmer). Following the final cycle, samples were incubated at $72^{\circ} \mathrm{C}$ for $20 \mathrm{~min}$ to ensure the completion of the final extension step. For reverse transcriptase (RT)-PCR analysis, RNA isolation was performed using Trizol (Gibco-Invitrogen Co.) according to the manufacturer's instructions. Total RNA was reverse transcribed and amplified using the EZ rTth RNA PCR Kit (Perkin Elmer) in a reaction mix as follows: $1 \times \mathrm{EZ}$ buffer, $300 \mu \mathrm{M}$ dNTPs, $5 \mathrm{U}$ rTth DNA polymerase, $2.5 \mathrm{mM} \mathrm{Mn}(\mathrm{OAc})_{2}, 0.5 \mu \mathrm{M}$ of primers pair specific for the Tax/Rex region of HTLV-1 [Kinoshita et al., 1989], or with primers specific for GAPDH as an internal control. Reverse transcription was performed at $55^{\circ} \mathrm{C}$ for $1 \mathrm{hr}$, followed by 35 cycles, each cycle consisting of $30 \mathrm{sec}$ at $94^{\circ} \mathrm{C}$ (DNA denaturation), $30 \mathrm{sec}$ at $55^{\circ} \mathrm{C}$ (primer annealing), and 45 sec at $72^{\circ} \mathrm{C}$ (primer extension) on a Cetus DNA thermal cycler 2400 (Perkin Elmer). Following the final cycle, samples were incubated at $72^{\circ} \mathrm{C}$ for $20 \mathrm{~min}$ to ensure the completion of the final extension step. Amplified DNA and RNA were analyzed by liquid hybridization, and probed using specific $\left[{ }^{32} \mathrm{P}\right]$-end-labeled oligonucleotides, as previously described [Mastino et al., 1997]. The samples were denatured for $5 \mathrm{~min}$ at $95^{\circ} \mathrm{C}$ and annealed at $55^{\circ} \mathrm{C}$ for $15 \mathrm{~min}$. A running buffer was added to the samples and they were loaded onto an $8 \%$ gel to detect the amplified products. Following electrophoresis the gel was then dried and exposed to Kodak XAR-5 film (Kodak Company, Rochester, NY) for autoradiography. For protein analysis by Western blotting, 5 million cells from each culture condition were rinsed three times in cold PBS and then solubilized in lysis buffer $(50 \mathrm{mM}$ Tris-HCl pH 7.4, 1 mM EDTA, 1 mM EGTA pH 7.4, 1\% Triton-X, $\mathrm{NaCl} 150 \mathrm{mM}, 0.25 \%$ sodium deoxycholate, $1 \%$ NP-40 and, freshly added, $1 \mathrm{mM}$ PMSF, $5 \mu \mathrm{M}$ DTT, $1 \mu \mathrm{g} /$ $\mathrm{ml}$ leupeptin, $1 \mu \mathrm{g} / \mathrm{ml}$ pepstatin, $2 \mu \mathrm{g} / \mathrm{ml}$ aprotinin, $1 \mathrm{mM}$ $\mathrm{Na}_{3} \mathrm{VO}_{4}, 20 \mathrm{mM} \mathrm{Na} 3$; all from Sigma) on ice and centrifuged at $10,000 \mathrm{~g}$. An aliquot of the supernatant was saved for protein concentration determination, and the rest was boiled in SDS sample buffer $(50 \mathrm{mM}$ Tris$\mathrm{HCl} \mathrm{pH} \mathrm{7.4,} 10 \mathrm{mM}$ DTT, 5\% SDS, 0.005\% bromophenol blue, $10 \%$ glycerol, all from Sigma). Seventy microgram of proteins were loaded onto a $10 \%$ SDS-polyacrylamide gel, subjected to electrophoresis, and transferred to PVDF membrane (Biorad, Hercules, CA), which was subsequently stained with $0.2 \%$ Ponceau red (Sigma) to ensure equal protein loading and transfer. After blocking the membrane in $10 \%$ non-fat dried milk (Biorad) and 3\% BSA in TTBS (20 mM Tris-HCl pH 7.4, $500 \mathrm{mM}$ $\mathrm{NaCl}, 0.15 \%$ Tween 20, all from Sigma), the blots were incubated overnight at $4{ }^{\circ} \mathrm{C}$ with $5 \mu \mathrm{g} / \mathrm{ml}$ of anti-TAX $\mathrm{mAb}$ (a generous gift from Elliot Cowan, FDA, Division of Transfusion-transmitted diseases, Bethesda, MD), with $1.5 \mu \mathrm{g} / \mathrm{ml}$ of anti-PARP $\mathrm{mAb}$ or with $1: 10,000$ dilution of anti-Caspase $3 \mathrm{mAb}$ (BD Biosciences, Pharmingen, San Diego, CA). Subsequently, the blots were washed three times in TTBS and then incubated with anti-mouse IgG (for primary mAb anti-PARP and anti-Tax, Biorad) or with anti-rabbit IgG (for primary $\mathrm{mAb}$ anti-caspase 3, Calbiochem) conjugated to peroxidase (Biorad). Binding of antibodies was detected by chemoluminescence staining using the ECL detection kit (Amersham Biosciences, Little Chalfont, UK). Analysis of Fas expression was performed by flow cytometry. Approximately $5 \times 10^{5}$ cells were diluted in $50 \mu \mathrm{l}$ of culture medium. A FITC-conjugated CD95 mAb (BD Biosciences, Pharmingen) was added and staining was performed at $4^{\circ} \mathrm{C}$ for $30 \mathrm{~min}$. Successively, samples were washed, fixed in $0.2 \%$ paraformaldehyde (Sigma) and analysis was performed using a FACScan flow cytometer. 


\section{Fluorescence In Situ Hybridization}

For fluorescence in situ hybridization (FISH), the pMT2 plasmid (a generous gift of G. Franchini, NCI, BRL, AMRSV, Bethesda, MD) was used to generate labeled fragments for probe. pMT2 is a pSP64 plasmid containing the entire HTLV-1 genome. Five microgram of purified HTLV-1 genome was digested in $50 \mu \mathrm{l}$ reaction volume of nick translation buffer containing $50 \mu \mathrm{M}$ dATP, dCTP, dGTP, $10 \mu \mathrm{M}$ dTTP, 10 mM DTT, $50 \mathrm{ng}$ bovine serum albumin, DNase I/DNA polymerase (all from Promega, Madison, WI) and $40 \mu \mathrm{M}$ digoxigenin11-dUTP (Roche Diagnostics). The reaction was carried out for $75 \mathrm{~min}$ at $15^{\circ} \mathrm{C}$ and was terminated by adding $50 \mathrm{mM}$ of EDTA. The DNA probe pool was precipitated by adding $0.3 \mathrm{M}$ of sodium acetate, ethanol $100 \%$, and salmon sperm $0.1 \mu \mathrm{g} / \mu \mathrm{l}$ (all from Sigma). An aliquot of reaction mix was loaded onto $0.7 \%$ of agarose gel to check the length of fragments. To promote cell adhesion, slides were treated with $5 \mu \mathrm{g} / \mathrm{cm}^{2}$ fibronectin (Roche Diagnostics) for $1 \mathrm{hr}$ at room temperature. After one wash with PBS, infected or uninfected cells were spread onto slides and incubated with $100 \mu \mathrm{g} / \mathrm{ml}$ RNase A (Sigma) in $2 \times \mathrm{SSC}$ for $1 \mathrm{hr}$ a $37^{\circ} \mathrm{C}$. Slides were then dehydrated in a graded ethanol series $(75,90$, and $100 \%$ for a few seconds), incubated with $10 \mu \mathrm{g} / \mathrm{ml}$ pepsin in $\mathrm{HCl} 10 \mathrm{mM}$ (Sigma) for $10 \mathrm{~min}$ at $37^{\circ} \mathrm{C}$, rinsed twice with $\mathrm{PBS}$ and once with PBS plus $50 \mathrm{mM} \mathrm{MgCl}_{2}$, fixed with $1 \%$ formaldehyde in PBS plus $50 \mathrm{mM} \mathrm{MgCl}_{2}$ and, finally, dehydrated. Aliquots of $80 \mathrm{ng}$ digoxigenin-labeled probes were layered onto each slide. The digoxigeninlabeled probe pool was resuspended in a hybridization mix containing $50 \%$ deionized formamide, $2 \times$ SSC, $10 \%$ dextran sulphate, and $50 \mathrm{mM}$ sodium phosphate (all from Sigma). Slides were denatured at $80^{\circ} \mathrm{C}$ for $4 \mathrm{~min}$ and hybridization was carried out in a humidified chamber overnight at $37^{\circ} \mathrm{C}$. After hybridization, slides were washed twice in $2 \times \mathrm{SSC}$ plus $50 \%$ deionized formamide for $2 \mathrm{~min}$ and twice in $2 \times \mathrm{SSC}$ for $2 \mathrm{~min}$, both at $42^{\circ} \mathrm{C}$. Slides were then incubated for $1 \mathrm{hr}$ in $1 \times$ blocking buffer (Roche Diagnostics) at room temperature to prevent non-specific antibody binding. To detect digoxigenin-labeled probes a fluorescence-based amplification system of the signal was used. The slides were incubated with a mouse IgG1 Mab specific for the hapten digoxigenin. Then, the first antibody was detected by a second Mab, anti- anti mouse-IgG1-digoxigenin and finally the slides were incubated with a FITC-conjugated anti-digoxigenin Ab (Fluorescent Antibody Enhancer Set for DIG Detection; Roche Diagnostics). The incubations with each antibody were carried out at $37^{\circ} \mathrm{C}$ for $1 \mathrm{hr}$. Slides were then washed in $2 \times \mathrm{SSC}$ plus $0.02 \%$ Tween 20. After dehydration in graded alcohol series, the nuclei were counterstained with HOECHST 33342 (Sigma) at a concentration of $10 \mathrm{ng} / \mathrm{ml}$ and, finally, the slides were covered with anti-fade solution and a cover slip and sealed with rubber cement. Slides were examined using a DMR fluorescence microscope and CDD camera equipped with $100 \times$ oil immersion lens (Leica, Solms, Germany). A minimum of 1,000 cells/condition was evaluated. For presentation, digital images were processed using Adobe Photoshop 5.0.

\section{Southern Blot Hybridization}

High molecular weight DNA was extracted from a culture infected in vitro with HTLV-I, frozen at different times after infection and thawed 1 week before DNA extraction, from MT-2 cells infected chronically as a monoclonal positive control, and from uninfected PBLs as a negative control. Aliquots of $50 \mu \mathrm{g}$ DNA were digested overnight at $37^{\circ} \mathrm{C}$ with $100 \mathrm{U}$ of restriction endonuclease Bam HI (Promega). The digested products were subjected to electrophoresis for $8 \mathrm{hr}$ at $50 \mathrm{~V}$ on $0.8 \%$ agarose, in TBE and transferred by vacuum blotter (Biorad) to positively charged membrane (BrigthStar Plus; Ambion, Austin, TX) pre-hybridized previously with $5 \times$ Denhardth solution, $6 \times \mathrm{SSC}, 0.5 \% \mathrm{SDS}$, and $1 \mu \mathrm{g} / \mathrm{ml}$ of salmon sperm DNA (all from Sigma) for $4 \mathrm{hr}$ at $42^{\circ} \mathrm{C}$. Membrane was then hybridized with a ${ }^{32} \mathrm{P}$-nicktranslated HTLV-1 probe, pMT-2, which detects the entire sequence of HTLV-1 (a generous gift from G. Franchini), overnight at $42{ }^{\circ} \mathrm{C}$ in $30 \%$ formamide, $6 \times \mathrm{SSC}, 0.01 \mathrm{M}$ EDTA, $1 \mu \mathrm{g} / \mathrm{ml}$ of salmon sperm DNA. After hybridization, the membrane was washed three times in 2-0.1 $\times$ SSC and 0.5-0.1\% SDS. Proviral digestion fragments were detected by autoradiography on Kodak film.

\section{Statistical Analysis}

Data analysis was performed using the SPSS statistical software system (version 10.0 for Windows, Chicago, IL). Comparisons of means between groups were carried out using the Student's $t$-test for independent samples.

\section{RESULTS}

\section{Apoptosis in Cell Cultures Infected Recently by HTLV-1}

Susceptibility to apoptosis of PBLs from different donors co-cultivated in vitro with irradiated MT-2 cells was evaluated at different times after infection, by DNA staining with propidium iodide. Figure $1 \mathrm{~A}$ shows the results obtained in duplicate cultures of PBLs, from three representative donors, at 4, 6, 8, and 14 weeks after infection, expressed as mean values $\pm \mathrm{SE}$ of spontaneous apoptosis. Infected cultures, grown in IL-2 as usual, showed levels of spontaneous apoptosis that were always quite elevated, with a high degree of individual variability in their amount. In particular, remarkable peaks of apoptosis that varied individually in extent and time of occurrence, were detectable in cultures shown in Figure 1A, as well as in most other cultures tested, during this relatively short time after infection. Nevertheless, after these peaks there was a general tendency towards stability of apoptosis at lower levels. Note that cell cultures underwent density-gradient centrifugation $24 \mathrm{hr}$ before apoptosis detection. Thus, apoptosis values 


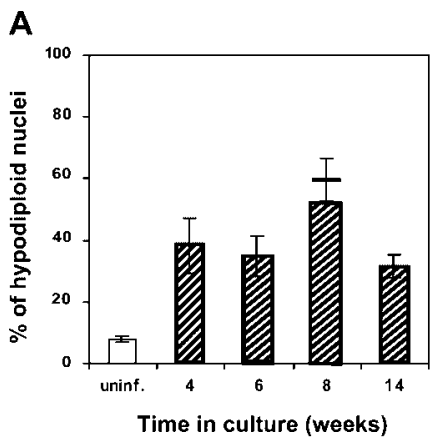

B
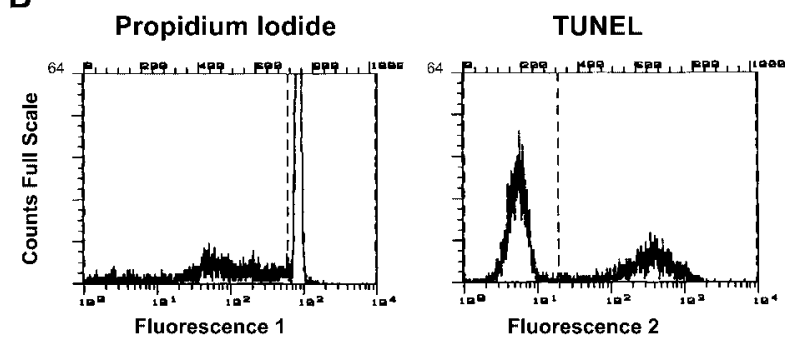

C
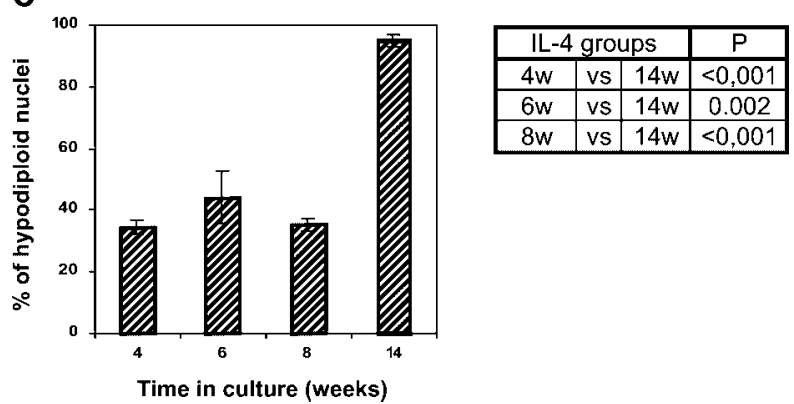

Fig. 1. A: Susceptibility to apoptosis of PBLs infected in vitro with HTLV-1 by coculture with irradiated MT-2 (dashed bars). Apoptosis was evaluated at $4,6,8$, and 14 weeks from infection in cultures of PBLs maintained in IL-2 and was detected, after $24 \mathrm{hr}$ incubation following the removal of debris and dead cells by density gradient centrifugation, by flow cytometry analysis of hypodiploid nuclei isolated and stained with a hypotonic solution containing detergent and propidium iodide. Results are expressed as means values \pm standard errors obtained from duplicate cultures from three different donors (total $n=6$ ). Results obtained in uninfected PBLs from ten donors cultured in the presence of IL-2 for 7 days, are also shown (open bar, uninf.). Statistical comparisons using Student's $t$-test for independent samples are reported as a small table on the right side of the panel. Omitted comparisons were not significant. B: Representative, comparative analysis of apoptosis by single-fuorescence flow cytometry of a culture of PBLs maintained in IL-2, at 6 weeks after HTLV-1 infection, using the propidium iodidide staining of nuclei technique (left panel) and the TUNEL assay (right panel). The dashed line in the left panel (propidium iodide) indicates the boundary among the peaks of diploid nuclei (right) and hypodiploid nuclei (left), which was arbitrarily set and used for evaluation of \% apoptosis (37\%). The dashed line in the right panel (TUNEL) indicates the boundary among the peaks of negative (left) and positive (right) apoptotic cells, which was arbitrarily set on control samples incubated with fluorescein isothiocyanate-dUTP solution alone without TdT (not shown), maintained for all other samples and used for evaluation of \% apoptosis $(36 \%)$. C. Susceptibility to apoptosis in the same cultures of PBLs illustrated in (A), infected in vitro with HTLV-1 by coculture with irradiated MT-2, but maintained in parallel with IL-4. Apoptosis was evaluated and results are expressed as described in (A). Statistical comparisons using Student's $t$-test for independent samples are reported as a small table on the right side of the panel. Omitted comparisons were not significant. refer to events accumulated mainly during the last $24 \mathrm{hr}$ and not during the entire period in culture. To exclude possible artefacts in detecting apoptosis, in some experiments the identification of apoptotic cells was also carried out by using the TUNEL technique. As an example, Figure 1B shows the analysis of apoptosis by the detection of hypodiploid nuclei stained with propidium iodide (left panel) in one of the cultures shown in Figure $1 \mathrm{~A}$ at 6 weeks after infection, in comparison with apoptosis detected by flow cytometry analysis of fluorescence relative to dUTP-FITC incorporation (TUNEL, right panel) in the same sample. Both techniques gave similar results for quantitative detection of apoptosis. As shown in Figure 1A, results refer to apoptosis detected in infected cultures starting at 4 weeks after infection, to exclude, without any doubt, the presence of residual MT-2 dead cells or of viral material from MT-2 donor cells, as previously shown by us [Mastino et al., 1997]. For comparison, the same Figure 1A shows the mean value $\pm \mathrm{SE}$ of spontaneous apoptosis detected in uninfected cultures from 10 donors, set up in duplicate. Uninfected PBLs were prepared similarly to infected cells and were cultured with the addition of the same low concentration of IL-2, without further stimulation, for 7 days before apoptosis was detected. This is a culture condition which was found suitable to represent physiologic, IL-2-dependent proneness to undergo apoptosis in non-stimulated PBLs. However, in uninfected non-stimulated cultures of PBLs, increasing levels of apoptosis were detected progressively due to exhaustion of cell growth and premature termination of culture within 6-7 weeks from the onset, as expected (data not shown). The use of uninfected, non-stimulated PBLs as a control suffered from the absence of continuous viral antigen stimulation and of high level proliferation in HTLV-1-infected cells. For this reason, to assess better the relationship between susceptibility to apoptotic cell death of HTLV-1infected PBLs in the first weeks after infection and their proneness to go towards immortalization, it was decided to measure levels of apoptosis also in HTLV-1 infected cultures maintained in parallel with the addition of IL-4 to the medium, as a further control. In was demonstrated previously that also this culture condition allowed the cells to be infected efficiently by HTLV-1, but only cells maintained in IL-2 went towards immortalization following HTLV-1 infection in vitro [Mastino et al., 1997]. Figure 1C shows the results of spontaneous apoptosis obtained in duplicate cultures of PBLs from the same three representative donors shown in Figure 1A, when grown in IL-4 at 4, 6, 8, and 14 weeks after infection. The results show that, even if $\mathrm{HTLV}-1$ infection of PBLs grown in IL-4 evidently prevented premature termination of cultures in comparison with uninfected cultures, after a few weeks during which levels of apoptosis were comparable to those observed in HTLV-1-infected PBLs maintained in IL-2, similarly to what observed for uninfected PBLs, there was an inexorable tendency towards a progressive increase in apoptosis levels, reaching values close to $100 \%$, before termination of the cultures. 
A

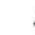

pol

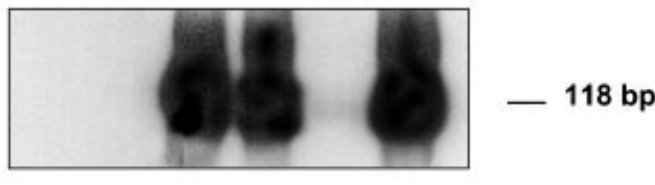

B

$\operatorname{tax} /$ rex

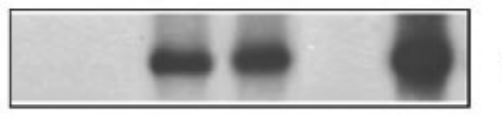

C

Tax

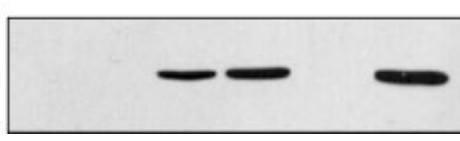

$-40 \mathrm{kD}$

Fig. 2. Detection of presence and expression of HTLV-1 in one representative culture of PBLs infected in vitro by exposure to irradiated MT-2 cell line, at 4 weeks after infection. A: Detection of proviral DNA by liquid hybridisation with a specific probe after PCR of $\mathrm{pol}$. B: Detection of tax/rex expression by liquid hybridization with a specific probe after RT-PCR. C: Detection of HTLV-1 protein Tax by Western blotting. Lane 1, uninfected PBLs kept in IL-2; lane 2, uninfected PBLs kept in IL-4; lane 3, HTLV-1-infected PBLs kept in IL-2; lane 4, HTLV-1-infected PBLs kept in IL-4; lane 5, irradiated MT-2; lane 6, viable MT-2. Similar results were obtained in cultures from four other donors tested.

Infection of PBLs in these experiments was directly confirmed by assessing the amount of proviral DNA and viral RNA, through DNA- and RT-PCR using primers specific for $p o l$ and the splicing region tax/rex, respectively, and by Western blot analysis using an anti-Tax mouse monoclonal antibody. Figure 2 shows the results obtained in a representative cell culture using these methods. As expected, the presence of proviral DNA of HTLV-1 and its expression, assayed as above-described, was equally detected in parallel cultures of PBLs exposed to irradiated MT-2 and cultured with the addition of IL-2 or IL-4 and, more remarkably, in viable MT-2 cells, but not in uninfected PBLs or irradiated MT2 alone. However, these assays give information on the infectivity status at entire population level, and not at single cell level. Thus, we asked ourselves whether apoptosis was actually detected in infected cells or rather whether it was related to a possible minority of uninfected cells, destined to die, still present in the cultures. To answer this question, some HTLV-1infected cultures, were simultaneously analyzed for the presence of proviral DNA and apoptosis by fluorescence microscopy at single cell level after 4 weeks from initiating the co-culture with irradiated MT-2. The viral genome was localized within the nuclei of infected cells cultures by FISH technique, using digoxigeninlabeled DNA probes derived from the p-MT-2 plasmid containing the entire HTLV-1 genome. Figure 3A shows the results obtained in a representative cell line infected with HTLV-1. Approximately $80 \%$ of the cells showed indubitable presence of HTLV-1 genome within Hoechst-counterstained nuclei, as demonstrated by the presence of an average of two, clear, green spots per nucleus. In contrast, uninfected PBLs grown for 2 weeks with IL-2 were completely negative for viral-DNA presence (Fig. 3B). Similar results were obtained in all the 6 infected and control cultures examined, with an average positive-cell value \pm SD of $86.3 \% \pm 3.6$ in infected cell cultures and $0.1 \% \pm 0.2$ positive cells in control, uninfected cells. Percentages of positive cells obtained in infected cultures were only slightly lower than those obtained in the chronically infected cell line HUT102, using the same technique (Fig. 3C, 92.9\% $\pm 2.0, \mathrm{n}=6$ ). Considering that the HUT102 cell line consists of $100 \%$ HTLV-1 infected cells, with most of the cells harboring four copies of the HTLV-1 genome, this indicates that the FISH technique we set up, even if extremely efficient in detecting viral-DNA with insignificant false-positive results, leads to a slight under-estimation of the infected cells. Consequently, based on the results, it can be assumed that nearly all the cells in cultures exposed to irradiated MT-2, according to our model of experimental infection, harbor HTLV-1 at 4 weeks after infection. Hoechst staining allowed us to detect also the morphological changes of nuclei associated with apoptosis. This analysis revealed that $27.1 \pm 5.3 \%$ of the total cells
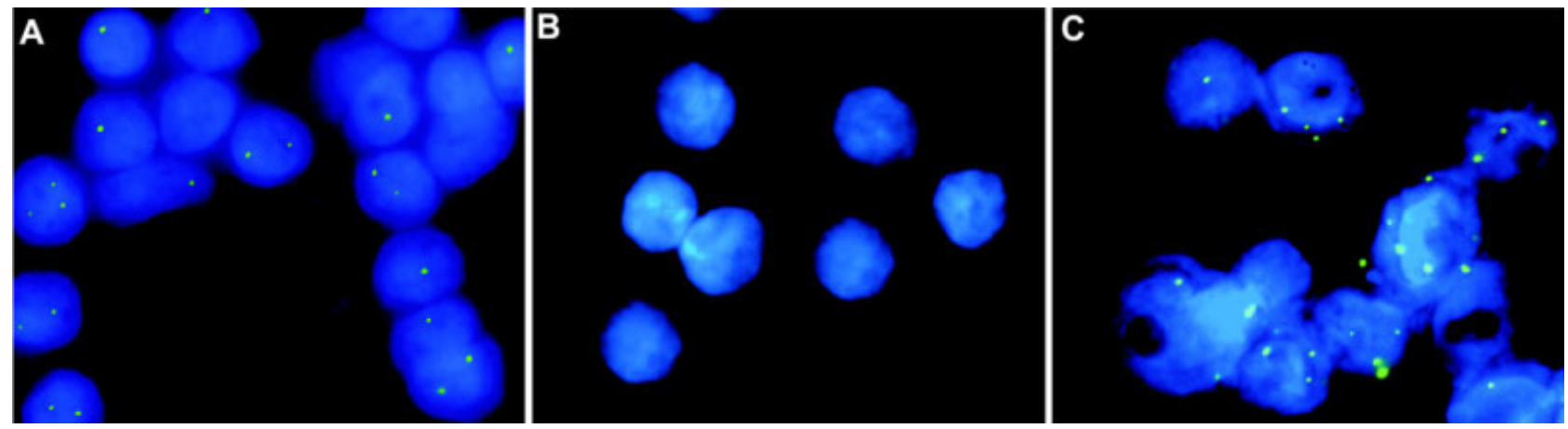

Fig. 3. Detection of HTLV-1 genome in Hoechst-counterstained nuclei from one representative culture of PBLs infected in vitro by exposure to irradiated MT-2 cell line, at 4 weeks after infection, using the FISH technique. Cells were dehydrated, fixed, and probed with a pool of digoxigenin-labeled fragments from HTLV-1 genome, reveal- ed by a fluorescence-based amplification system, as described in "Materials and Methods" (green spots). A: HTLV-1-infected PBLs culture, maintained in IL-2, at 4 weeks after infection. B: Uninfected PBLs culture maintained in IL-2 for 2 weeks. C: The chronically infected HUT 102 cell line. 

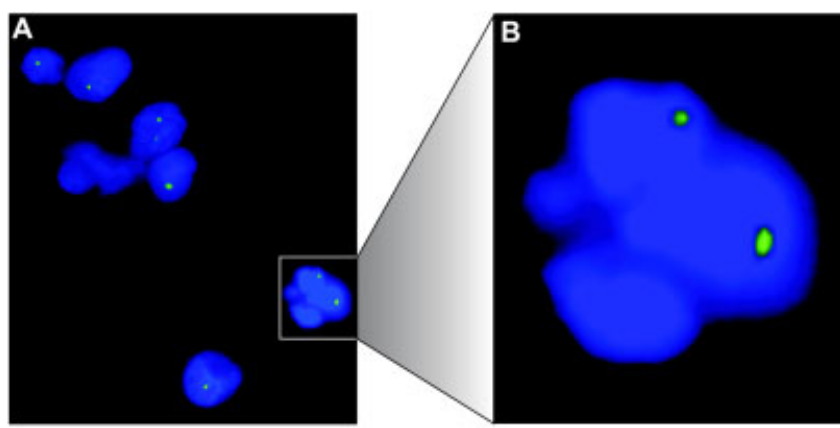

Fig. 4. Detection of double positive HTLV-1-DNA/apoptosis nuclei. A: Cells from one representative HTLV-1-infected culture maintained in IL-2 for 4 weeks were treated and stained for the FISH-technique and Hoechst counterstaining of nuclei as described in Figure 3 and "Materials and Methods;" green spots indicate HTLV-1-positive nuclei. B: Particular of an isolated nucleus showing both positivity for HTLV-IDNA (two green spots, presumably two copies of integrated HTLV-1 genome) and the characteristic morphology of an apoptotic nucleus.

exhibited morphological characteristics of apoptosis, with nuclei showing fragmentation or aggregation of chromatin at the periphery. Combined FISH/Hoechststaining analysis also showed that, among the HTLV-1proviral-DNA positive cells, an average percentage of $28.9 \pm 5.2 \%$ of the cells were also apoptotic. Figure $4 \mathrm{~A}$ illustrates the appearance of HTLV-1/apoptosis doublepositive nuclei in one representative culture. In particular, Figure 4B shows an isolated HTLV-1-positive cell whose nucleus exhibits the typical feature of apoptosis. Thus, apoptosis was observed with the same frequency in the entire population as well as in the cells in which proviral DNA could be detected. In other experiments, using two-fluorescence flow cytometry analysis, apoptosis was detected at similar levels in cells expressing the viral core protein p19 as well as in cells negative for p19 expression (data not shown). Taken together, these results indicate that provirus presence or its expression in cells infected by HTLV-1 in vitro did not confer protection against apoptosis in the short/medium term, but rather coexisted with elevated levels of apoptotic cell death in the first weeks after infection.

\section{The Relationship Between Apoptosis and Cell Growth in Separated CD4+ Cells Infected by HTLV-1}

To obtain further information on the relationship between the susceptibility to cell death of HTLV-1infected PBLs and their proneness to grow extensively, in other experiments levels of apoptosis and cell growth were detected in parallel cultures of isolated CD4+ Tcells infected in vitro with HTLV-1 and maintained alternatively with the addition of IL-2 or IL-4 to the medium. Figure 5 shows the variations in the levels of apoptosis and cell growth at 2, 4, 6, 8, and 12 weeks post infection. The results indicate that there was a clear tendency towards a progressive increase in the ratio between cell growth and apoptosis in cultures kept in IL2 while this ratio decreased in infected cultures kept in IL-4. Results were similar to those obtained in cultures of total PBLs infected in vitro with HTLV-1. However,

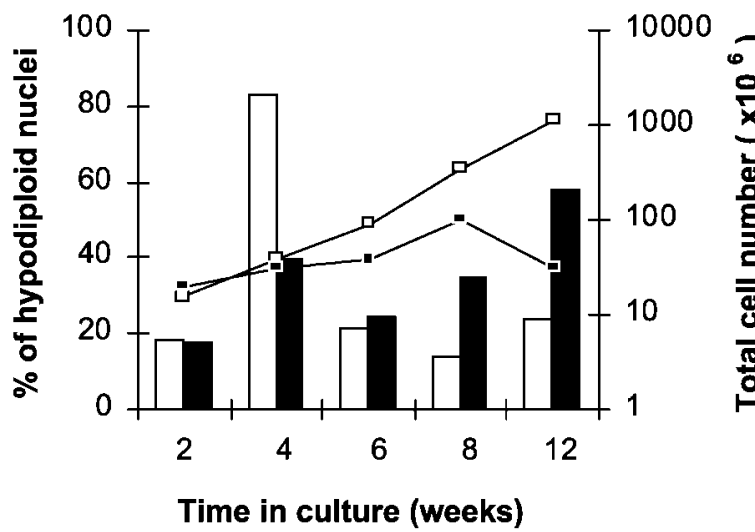

Fig. 5. Comparison of cell growth and apoptosis in HTLV-1-infected cultures of separated CD4+ cells maintained in IL-2 or in IL-4. Levels of apoptosis (bars) and cell growth (lines) in parallel cultures set up from cells from the same donor and maintained in IL-2 (open) or IL-4 (solid), at $2,4,6,8$, and 12 weeks after HTLV-1 infection. Apoptosis was detected, after $24 \mathrm{hr}$ incubation following the removal of debris and dead cells by density gradient centrifugation, by flow cytometry analysis of hypodiploid nuclei isolated and stained with a hypotonic solution containing detergent and propidium iodide; cell growth was measured by detection of the number of viable cells using the trypan blue assay and is expressed as a total cell number, calculated theoretically as described in "Materials and Methods."

cultures of separated CD4+ cells showed an anticipation in the peak of apoptosis and in the successive start of the exponential phase of cell growth, characteristic of IL-2driven immortalization of infected cells, in comparison with total PBLs.

\section{Spontaneous and Fas-Mediated Apoptosis in the Long-Term Cultures Infected by HTLV-1 In Vitro}

Spontaneous apoptosis and the response to anti-Fasinduction of apoptosis was also evaluated in a number of cultures of PBLs infected in vitro by HTLV-1 and maintained in the long-term, in comparison with values observed in short-term cultures. The response to antiFas was expressed as an induction index calculated for each culture as follows:

\section{induction index $=\%$ apoptosis with anti-Fas $/ \%$ spon- taneous apoptosis.}

Cultures, grown in IL-2, were evaluated at 2, 6, 24, and 54 weeks after HTLV-1 infection in vitro. The results show a tendency towards decreasing values of spontaneous apoptosis in the long-term period (Fig. 6A). Conversely, infected cultures did not show the same decreasing tendency in the response to Fas-mediated apoptosis in the long-term period (Fig. 6B). This effect was the result of both a fairly low level of spontaneous apoptosis in older cultures (Fig. 6A) and the maintenance of elevated sensitivity to anti-Fas response in HTLV-1 infected cells during the culture. Moreover, to know whether the shift towards a phenotype with lower levels of spontaneous apoptosis in the long-term period in our cultures was the consequence of a monoclonal selection, experiments were undertaken using Southern blotting analysis following proviral digestion by a restriction endonuclease, in a representative HTLV-1 infected 
A

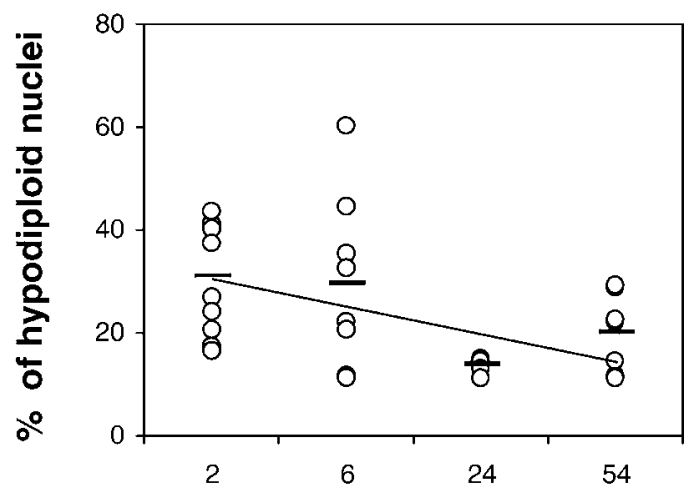

Time in culture (weeks)

B

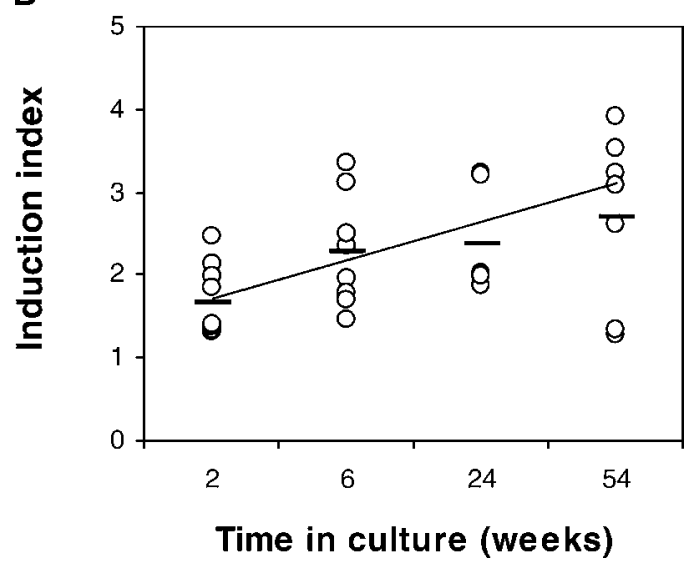

Fig. 6. Spontaneous and Fas-mediated apoptosis in long-term cultures of PBLs infected in vitro with HTLV-1. A: Spontaneous apoptosis detected by flow cytometry analysis of hypodiploid nuclei, $24 \mathrm{hr}$ after debris and dead cell removal by density gradient centrifugation, following detergent treatment and staining with propidium iodide, in cultures of PBLs infected in vitro with HTLV-1, at 2, 6, 24, and 54 weeks after infection. Individual values (circles), means values at each time and regression line are shown. Statistical comparison by Student's $t$-test for independent samples gave the following results: week 2 vs. week $24, P=0.001$; week 2 vs. week $54, P=0.028$; week 6 vs. week $24, P=0.031$. B: Response to apoptosis induced by agonist antiFas antibody, expressed as induction index calculated as the ratio between anti-Fas-induced apoptosis and spontaneous apoptosis detected by flow cytometry analysis of hypodiploid nuclei, following detergent treatment and staining with propidium iodide, in the same cultures of PBLs infected in vitro with HTLV-1 shown in panel A, at 2, 6 , 24 , and 54 weeks after infection. Individual values (circles) and mean values at each time are shown. Statistical comparison by Student's $t$ test for independent samples gave the following results: week 2 vs. week $6, P=0.045$; week 2 vs. week $24, P=0.049$; week 2 vs. week 54 , $P=0.037$

cell line grown in IL-2. Samples frozen at different times after infection, were thawed and cultured for 1 week before removing debris and testing proviral integration as well as apoptosis. Results showed patterns of multiple integration sites (diffuse smear), without any appreciable difference at 5 weeks, 6 months and 19 months after infection (data not shown), while percentages of apoptosis at the latter times were about half that observed at 5 weeks after infection $(36,20$, and $17 \%$, at 5 weeks, 6 months, and 19 months, respectively). Conversely, samples from MT-2 cells showed an incontrovertible pattern of a single integration site (single band).
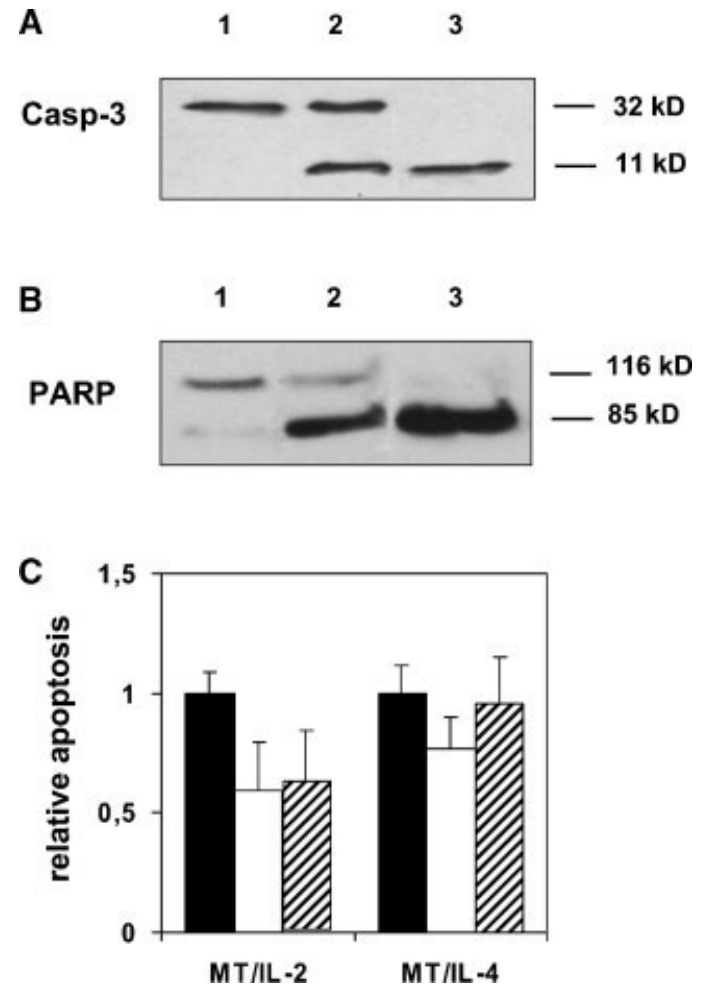

Fig. 7. Caspase cascade in cultures of PBLs infected in vitro by HTLV-1. A: Western blotting analysis of caspase 3, in one representative culture of three analyzed, at 4 weeks after HTLV-1 infection Caspase 3 was detected as the proenzyme $(32 \mathrm{kD})$ and as the activated form $(11 \mathrm{kD})$. Lane 1, uninfected PBLs grown in IL-2; lane 2, infected PBLs grown in IL-2; lane 3, infected PBLs grown in IL-4. B: Western blotting analysis of PARP, in one representative culture of three analyzed, at 4 weeks after HTLV-1 infection. PARP was detected as an active or an inactivated form (116 and $85 \mathrm{kD}$, respectively). Lane 1, uninfected PBLs grown in IL-2; lane 2, infected PBLs grown in IL-2; lane 3, infected PBLs grown in IL-4. C: Inhibition of apoptosis by caspase inhibitors in cultures of PBLs maintained in IL-2 or IL-4, at 4 weeks from HTLV-1 infection. The results are expressed as mean relative apoptosis \pm standard deviation, calculated with respect to mean values obtained in diluent-treated control samples within the experiment. Data represent the cumulative results obtained from at least duplicate samples for each experimental condition, in three independent experiments. Control PBLs (solid bars), Z-VAD-FMKtreated PBLs (bars), Z-DEVD-FMK-treated PBLs (dashed bars). Statistical comparison by Student's $t$-test for independent samples gave the following results: Z-VAD-FMK-treated grown in IL-2 vs. diluent-treated grown in IL-2, $P=0.003$; Z-DEVD-FMK-treated grown in IL-2 vs. diluent-treated grown in IL-2, $P=0.006$; Z-DEVD-FMKtreated grown in IL-2 vs. Z-VAD-FMK-treated grown in IL-2, NS; ZVAD-FMK-treated grown in IL-4 vs. diluent-treated grown in IL-4, $P=0.005$; Z-DEVD-FMK-treated grown in IL-4 vs. diluent-treated grown in IL-4, NS; Z-DEVD-FMK-treated grown in IL-4 vs. Z-VADFMK-treated grown in IL- $4, P=0.026$.

\section{Association With Caspase 3 Activation and PARP Cleavage and Lack of Evidence for Soluble Mediators in Apoptosis Detected in HTLV-1- Infected Cultures Destined to Immortalization}

Next, it was investigated whether apoptosis in HTLV1-infected cultures, kept in IL-2 or in IL-4, respectively was associated with the classical activation of downstream signals leading to cell death. Figure 7A shows that, at 4 weeks after infection in a representative experiment, both the pro-enzyme $(32 \mathrm{kD})$ and the cleavage fragment $(11 \mathrm{kD})$ of caspase 3 were detectable in 
samples from infected PBLs grown in IL-2, while those kept in IL-4 showed only the cleaved form. Caspases are able to cleave a number of different substrates; and it was shown that Poly(ADP-ribosyl) protein (PARP), a key effector in DNA repair, replication, and transcription, whose cleavage is directly implicated in the apoptosis execution phase, was highly inactivated. In fact, PARP was detected in HTLV-1-infected cultures maintained in IL-2 or in IL-4 at 4 weeks post infection in the $85 \mathrm{kD}$ inactivated form, as shown in Figure 7B. Conversely, uninfected control PBLs grown in IL-2 showed only the non-cleaved forms of both caspase 3 and PARP. It was then asked whether apoptosis in HTLV-I infected cells was affected by inhibition of caspase activity. Figure $7 \mathrm{C}$ shows the cumulative results of three independent experiments in which Z-VAD-FMK (pan-caspase) and Z-DEVD-FMK (caspase 3 specific) cell-membrane permeable caspase inhibitors, were added to at least duplicate samples from HTLV-1 infected cultures grown in either IL-2 or IL-4. The results are expressed as means \pm SD relative apoptosis with respect to control samples. Relative apoptosis was calculated, for each sample, as follows:

relative apoptosis $=\%$ apoptosis of the sample/mean $\%$ apoptosis from control samples treated with diluent alone within the same experiment.

The results show that Z-VAD-FMK significantly, even if only partly, inhibited apoptosis detected in HTLV-1infected cultures grown in IL-2 as well as in those grown in IL-4. Conversely, Z-DEVD-FMK inhibited significantly apoptosis in infected samples grown in IL-2, but not in those grown in IL-4. Moreover, Z-DEVD-FMK was significantly less effective than Z-VAD-FMK in samples grown in IL-4, but not in those grown in IL-2. These results confirm the involvement of the caspase cascade in apoptosis detected in HTLV-1 infected cultures. Moreover, experiments using Z-DEVD-FMK suggest that caspase 3 plays a major role in apoptosis in PBLs grown in IL-2, destined to HTLV-1-driven immortalization. Conversely, caspases other than caspase 3 seem to contribute to the execution phase of apoptosis in infected PBLs maintained in IL-4, destined to exhaustion of cell growth. One possible mechanism involved in apoptosis in infected cultures could be the production of death-inducing soluble factors by infected cells. To shed light on this point, supernatants were collected from HTLV-1 infected cultures at various times and assayed: (1) the amount of Fas-Ligand by ELISA, and (2) the pro-apoptotic activity of the supernatants using the death-receptor sensitive MOLT-3 cell line, as target cells. For comparison, supernatants obtained from PHA-stimulated or non-stimulated uninfected PBLs were also assayed as a control. However, the results were completely negative: neither amounts of Fas-Ligand detected in supernatants from infected cultures nor apoptosis detected in MOLT-3 cells exposed to the same supernatants were over the levels of controls (data not shown). Altogether these results suggest that the involvement of soluble mediators in the high level of apoptosis detected in HTLV-1-infected cultures can be reasonably excluded.

\section{DISCUSSION}

In the present study the proneness to apoptosis of PBLs was investigated from normal donors de novo infected in vitro with HTLV-1. Results have shown that HTLV-1 infection induced in the long-term, as expected, a sustained growth in the recipient cells. However, unexpectedly the tendency towards an uncontrolled expansion of HTLV-1 infected PBLs was counteracted, especially during the first weeks after infection, by a remarkable level of proneness to apoptosis. Relevant to this point it is worthwhile highlighting that our results clearly demonstrate that elevated levels of apoptotic cell death observed in the first weeks after in vitro infection were not related to the presence of residual uninfected cells destined, in the absence of stimulation, to death by exhaustion of growth. Rather, it was demonstrated that the majority of the cells in which an HTLV-I/DNA integration within the cellular genome efficiently occurred were relentlessly destined to apoptosis early after infection. Even the expression of the retroviral genome did not confer particular protection against cell death, as demonstrated by experiments using p19 expression. Taken together these results suggest that, during the initial phase following in vitro infection of PBLs (414 weeks), a push towards apoptosis should largely prevail versus a predictable apoptosis inhibitory effect of the virus. Only successively, a relative decrease in apoptosis levels was normally observed in cultures of PBLs infected in vitro by HTLV-1 and kept, as usual, in IL-2. This decrease occurred generally in concomitance with a remarkable and irrepressible rise in cell counts. Conversely, cultures maintained in IL-4, that became infected but not immortalized by HTLV-1, were characterized by progressive, increasing levels of apoptosis leading to exhaustion of cell growth after a few weeks in culture. Thus, apparently, modulation of apoptosis plays a crucial role in the balance between cell growth and cell death that leads to the process of immortalization of PBLs in vitro by HTLV-1. HTLV-1 transformed cells in the long-term were found to be resistant to $\mathrm{TNF} \alpha$ - or Fas-induced apoptosis independently of TNF or Fas receptor expression [Kongphanich et al., 2002; Yang et al., 2002]. In particular, it was shown that resistance to Fas-mediated apoptosis in HTLV-1-infected cell lines might be dependent on the failure of Fas receptor clustering and caspase- 8 activation, and that this could be overcome by treatment with cycloheximide [Kongphanich et al., 2002]. In addition Fas resistance in HTLV-1transformed cells has been proposed to be irreversible owing to mutations in Fas-encoding genes, based on results obtained in a few adult T-cell leukemia/lymphoma cell lines [Tamya et al., 1998; Maeda et al., 1999]. This latter aspect might also be associated with a poor response to anticancer drugs [Tamya et al., 1998]. In agreement with these results, it was observed that MT-2 
transformed cells, although highly expressed Fas, were not susceptible to anti-Fas induced apoptosis (data not shown). However, the results also show that cultures of PBLs infected in vitro by HTLV-1, while reducing progressively their proneness to spontaneous apoptosis when progressing towards immortalization, maintained their susceptibility to Fas-mediated apoptosis for a considerable length in time. This should indicate that apoptosis mediated by Fas did not act as a selective mechanism for the outgrowth of immortalized HTLV-1infected cells, at least for many months in culture. In contrast, it has been shown that induction of Tax expression was associated with a remarkable upregulation of Fas-ligand at the transcriptional level [Chen et al., 1997]. Nevertheless, the production of Fasligand by cells infected with HTLV-1 in vitro Maeda et al. [1999]; in our experimental system was very low. Thus, it is highly improbable that the Fas/Fas-ligand system could be an upstream signal for the apoptotic pathway of infected cells in our HTLV-1-infected cultures. Moreover, apoptosis occurring in PBLs infected by HTLV-1 in vitro, grown either in IL-2 or IL-4, was relatively caspase-dependent, as shown by experiments using caspase inhibitors. However, experiments using the caspase-3 inhibitor indicate a difference between the apoptotic machinery activated in cells destined to immortalization by HTLV-1 and that acting in those, grown in IL-4, destined to inexorable death following infection, suggesting that caspase-3 plays a major role in apoptosis only in the former. Based on the results, the cascade of signals underlying cell death in HTLV-1-infected cells is still greatly unclear and further experiments should clarify these aspects. In addition, it is not possible to distinguish the contributions of the two, not necessarily mutually-exclusive, mechanisms which could reasonably play a role in the initially high proneness to apoptosis of cells infected by HTLV-I in vitro, i.e. (i) the direct activation of death pathways by HTLV-I using specific viral products and (ii) the alerting of innate or even adaptative host cell defense against viral infection. In fact, infected cells could either trigger cellular signals of stress-response to viral infection by activating their own mechanism of programmed cell death or be targeted by HTLV-1-specific cytotoxic effectors cells activating a fratricide apoptotic killing of infected cells in culture. Also environmental factors might affect the final fate of infected cells, as shown by our experiments using IL-4 to replace IL-2 as a growth factor. Progression towards exhaustion of the culture by total apoptotic cell death in infected cells maintained in IL-4, could be due to the inability of this cytokine to counteract pro-apoptotic signals consequent to infection and to promote survival of HTLV-1-infected T-cells. Conversely, the addition of IL-2 could allow the growth of HTLV-1-infected cultures by activating transduction pathways associated with IL-2-receptor signaling, such as JAK/STAT, that have been shown to be activated constitutively in adult T-cell leukemia/ lymphoma cells [Migone et al., 1995]. Thus, IL-2 could confer to some infected cells pro-survival characteristics which result in the selection of cells with a low proneness to apoptosis. This could represent the optimal condition allowing the virus, under the constant and persistent selective pressure of simultaneous virus- and/or celloriginated both pro-apoptotic and anti-apoptotic signals, favoring the cells able to better resist to apoptosis and respond to pro-survival signals. Relevant to this, it is worth noting that low proneness to spontaneous apoptosis in long-term cultures in comparison with cultures infected recently was the result of a polyclonal, and not yet monoclonal, IL-2-dependent expansion of HTLV-1infected PBLs, as suggested by Southern blotting experiments. A recent publication reports the gene-expression profiles of HTLV-1 transformed cells which sustain that virus transformation is associated to the deregulation of several anti- and pro-apoptotic genes [Pise-Masison et al., 2002]. In the same article it is considered that it is critical to elucidate which apoptotic genes are regulated at an early phase of viral transmission in order to understand the alteration occurring in the immortalization/transformation phase. Our novel data on de novo infection in vitro by HTLV-1 suggest that in the early phase and in the successive immortalization phase of infection, pro- or anti-apoptotic genes may be differently regulated by virus infection and by factors present in the milieu. Taken together these results suggest that HTLV-1 integration and expression are associated both with induction and inhibition of apoptosis and that the disruption of the balance between these two events in the different phases of infection regulates the immortalization of infected cells in long-term culture.

\section{ACKNOWLEDGMENTS}

We wish to thank Alison Inglis, BA, for her linguistic assistance.

\section{REFERENCES}

Barmak K, Harhaj E, Grant C, Alefantis T, Wigdahl B. 2003. Human T cell leukemia virus type I-induced disease: Pathways to cancer and neurodegeneration. Virology 308:1-12.

Brauweiler A, Garrus JE, Reed JC, Nyborg JK. 1997. Repression of Bax gene expression by the HTLV-1 Tax protein: Implications for suppression of apoptosis in virally infected cells. Virology 231:135-140.

Cavrois M, Wain-Hobson S, Gessain A, Plumelle Y, Wattel E. 1996. Adult T-cell leukemia/lymphoma on a background of clonally expanding human T-cell leukemia virus type-1 positive cells. Blood 88:4646-4650.

Chen X, Zachar V, Zdravkovic M, Guo M, Ebbesen P, Liu X. 1997. Role of Fas/Fas ligand pathway in apoptotic cell death induced by the human T cell lymphotropic virus type I Tax transactivator. J Gen Virol 78:3277-3285.

Chlichlia K, Moldenhauer G, Daniel PT, Busslinger M, Gazzolo L, Schirrmacher V, Khazaie K. 1995. Immediate effects of reversible HTLV-1 tax function: T-cell activation and apoptosis. Oncogene 10:269-277.

Chlichlia K, Busslingher M, Peter ME, Walczak H, Krammer PH, Schirrmacher V, Khazaie K. 1997. ICE-proteases mediate HTLV-I Tax-induced apoptotic T-cell death. Oncogene 14:2265-2272.

Copeland KFT, Haaksma AGM, Goudsmit J, Krammer PH, Heeney JL. 1994. Inhibition of apoptosis in T cells expressing human $\mathrm{T}$ cell leukaemia virus type I. AIDS Res Hum Retrov 10:1259-1267.

Hanahan D, Weinberg RA. 2000. The hallmarks of cancer. Cell 100:5770.

Kawakami A, Nakashima T, Sakai H, Urayama S, Yamasaki S, Hida A, Tsuboi M, Nakamura H, Ida H, Migita K, Kawabe Y, Eguchi K. 1999. Inhibition of caspase cascade by HTLV-1 Tax through induction of Nf-kB nuclear translocation. Blood 94:3847-3854. 
Kawata S, Ariumi Y, Shimotohno K. 2003. p21Waf1/Cip1/Sdi1 prevents apoptosis as well as stimulates growth in cells transformed or immortalized by human T-cell leukemia virus type 1encoded Tax. J Virol 77:7291-7299.

Kinoshita T, Masanori S, Tobinai K, Ito M, Ito S, Ikeda S, Tajima K, Shimotohno K, Sugimura T. 1989. Detection of mRNA for the tax1/ Rex1 gene of human T-cell leukemia virus type I in fresh peripheral blood mononuclear cells of adult T-cell leukemia patients and viral carriers by using polymerase chain reaction. Proc Nat Acad Sci 86:5620-5624.

Kongphanich A, Hieda M, Kurokawa K, Murata T, Kobayashi N. 2002. Overcoming the blockade at the upstream of caspase cascade in Fasresistant HTLV-I-infected T cells by cycloheximide. Bioch Biophys Res Comm 294:714-718.

Macchi B, Graziani G, Zhang J, Mastino A. 1993. Emergence of doublepositive CD4/CD8 cells from adult peripheral blood mononuclear cells infected with human T cell leukemia virus type I (HTLV-I). Cell Immunol 149:376-389.

Maeda T, Yamada Y, Moriuchi R, Shugahara K, Tsuruda K, Joh T, Atogami S, Tsukasaki K, Tomonaga M, Kamihira S. 1999. Fas gene mutation in the progression of Adult T cell leukaemia. J Exp Med 189:1063-1071.

Maruyama K, Fukushima T, Kawamura K, Mochizuki S. 1990. Chromosome and gene rearrangements in immortalized human lymphocytes infected with human T-lymphotropic virus type I. Cancer Res 50:5697S-5702S.

Mastino A, Grelli S, Favalli C, Matteucci C, De Carli M, Garaci E, Macchi B. 1997. Interleukin 4 stimulates infection and temporary growth of human neonatal lymphocytes exposed in vitro to human $\mathrm{T}$ lymphotropic virus type I, but fails to substitute for interleukin 2 in the immortalization of infected cultures. J Gen Virol 78:2565-2574.

Matteucci C, Grelli S, De Smaele E, Fontana C, Mastino A. 1999. Identification of nuclei from apoptotic, necrotic and viable lymphoid cells by using multiparameter flow cytometry. Cytometry 35:145-153.

Migone TS, Lin JX, Cereseto A, Mulloy JC, O'Shea JJ, Franchini G, Leonard WJ. 1995. Constitutively activated Jak-Stat pathway in T cells transformed with HTLV-1. Science 269:79-81.

Miyake H, Suzuki T, Hirai H, Yoshida M. 1999. Trans-activator Tax of human T-cell leukaemia virus type I enhances mutation frequency of the cellular genome. Virology 253:155-161.
Miyoshi I, Kubonishi I, Yoshimoto S, Akagi T, Ohtsuki Y, Shirashi Y Nagata K, Hinuma Y. 1981. Type C virus particles in a cord T-cell line derived by cocultivating normal human cord leucocytes and human leukemic cells. Nature 294:770-771.

Mori N, Fujii M, Cheng G, Ikeda S, Yamasaki Y, Yamada Y, Tomonaga S, Yamamoto N. 2001. Human T-cell leukaemia virus type 1 Tax induces the expression of anti-apoptotic gene Bcl-xl in human T-cell through nuclear factor-kB and c-AMP responsive element binding protein pathways. Virus Gene 22:279-287.

Mortreux F, Gabet A-S, Wattel E. 2003. Molecular and cellular aspects of HTLV-1 associated leukemogenesis in vivo. Leukemia 17:2638

Nicot C, Harrod R. 2000. Distinct p300-responsive mechanisms promote caspase-dependent apoptosis by human T-cell lymphotropic virus type 1 Tax protein. Mol Cell Biol 20:8580-8589.

Pise-Masison C, Radonovich M, Mahieux R, Chatterjee P, Whiteford C, Duvall J, Guillerm C, Gessain A, Brady JN. 2002. Transcription profile of cells infected with human T-cell leukemia virus type I compared with activated lymphocytes. Cancer Res 62:35623571

Ruckes T, Saul D, Van Snick J, Hermine O, Grassmann R. 2001. Autocrine antiapoptotic stimulation of cultured adult T-cell leukaemia cells by overexpression of the chemokine I-309. Blood 98:11501159 .

Tamya S, Etoh K, Suzushima H, Takatsuki K, Matsuoka M. 1998. Mutation of CD95 (Fas/Apo-1) gene in adult T-cell leukemia cells. Blood 91:3935-3942.

Torgeman A, Ben-Aroya Z, Grunspan A, Zelin E, Butovsky E, Hallak M, Lochelt M, Flugel RM, Livneeh E, Wolfson M, Kedar I, Aboud M. 2001. Activation of HTLV-I long terminal repeat by stress-inducing agents and protection of HTLV-I-infected T-cells from apoptosis by the viral Tax protein. Exp cell Res 271:169-179.

Tsuda H, Huang RW, Takatsuki K. 1993. Interleukin-2 prevent programmed cell death in adult T-cell leukaemia cells. Jpn J Cancer Res 84:431-437.

Yang Y, Hsu TY, Lin R-H, Su IJ, Chen JY, Yang CS. 2002. Resistance to tumor necrosis factor- $\alpha$-induced apoptosis in Human T-lymphotropic virus type I-infected T-cell lines. Aids Res Hum Retrov 18:207-212. 